\title{
Inactivation of the Phosphatase CheZ Alters Cell-Surface Properties of Azorhizobium caulinodans ORS571 and Symbiotic Association with Sesbania rostrata
}

\author{
Xiaolin Liu ${ }^{1,2,3}$ and Zhihong Xie ${ }^{1,3,+}$ \\ ${ }^{1}$ Key Laboratory of Coastal Biology and Bioresource Utilization, Yantai Institute of Coastal Zone Research, Chinese Academy of \\ Sciences, Yantai, People's Republic of China \\ ${ }^{2}$ University of Chinese Academy of Sciences, Beijing, People's Republic of China \\ ${ }^{3}$ Center for Ocean Mag-Science, Chinese Academy of Sciences, Qingdao, People's Republic of China
}

Accepted 8 July 2019.

\begin{abstract}
Azorhizobium caulinodans can form root and stem nodules with the host plant Sesbania rostrata. The role of the CheZ phosphatase in the $A$. caulinodans chemotaxis pathway was previously explored using the nonchemotactic cheZ mutant strain (AC601). This mutant displayed stronger attachment to the root surface, enhancing early colonization; however, this did not result in increased nodulation efficiency. In this study, we further investigated the role of $\mathrm{CheZ}$ in the interaction between strain ORS571 and the roots of its host plant. By tracking longterm colonization dynamic of cheZ mutant marked with LacZ, we found a decrease of colonization of the cheZ mutant during this process. Furthermore, the cheZ mutant could not spread on the root surface freely and was gradually outcompeted by the wild type in original colonization sites. Quantitative reverse-transcription PCR analyses showed that exp genes encoding exopolysaccharides synthesis, including oac3, were highly expressed in the cheZ mutant. Construction of a strain carrying a deletion of both $c h e Z$ and oac 3 resulted in a mutant strain defective in the colonization process to the same extent as found with the oac 3 single-mutant strain. This result suggested that the enhanced colonization of the cheZ mutant may be achieved through regulating the formation of exopolysaccharides. This shows the importance of the chemotactic proteins in the interaction between rhizobia and host plants, and expands our understanding of the symbiosis interaction between rhizobium and host plant.
\end{abstract}

Keywords: genetics and gene regulation, rhizobium-legume symbiosis

${ }^{\dagger}$ Corresponding author. Z. Xie; zhxie@yic.ac.cn

Funding: This work was financed by the National Natural Science Foundation of China (31570063, 31870020, and NSFC-Shandong Joint Fund Key Project U1806206), the Key Deployment Project of the Chinese Academy of Sciences (KFZD-SW-112), and Agricultural Scientific and Technological Innovation Project of Shandong Academy of Agricultural Sciences (+CXGC2016B10).

*The $\boldsymbol{e}$-Xtra logo stands for "electronic extra" and indicates that four supplementary figures and one supplementary table are published online.

The author(s) declare no conflict of interest.

๑) 2019 The American Phytopathological Society
Rhizobia can fix atmospheric nitrogen and promote plant growth and, in turn, plants can exudate various carbon and nitrogen sources, including sugar, amino acids, organic acids, and other compounds which provide nutrition and a suitable niche for rhizobia (Kamilova et al. 2006).

Azorhizobium caulinodans ORS571 was isolated from the stem of the tropical legume Sesbania rostrata (Dreyfus et al. 1988). This strain can induce nitrogen-fixing nodules on both the roots and the stems of the host. A. caulinodans can also fix nitrogen in a free-living state. Nodule formation of stems proceeds via crack entry at sites corresponding to predetermined primordia (Fernández-López et al. 1998; Tsien et al. 1983). For root nodulation, two modes of infection are employed by A. caulinodans: root hair invasion and intercellular invasion at the lateral root base. Root hair invasion is the best-known mode of invasion used in many model legumes such as Lotus japonica and Medicago truncatula (Goormachtig et al. 2004), while crack entry infection at lateral root base is found in some tropical legumes, including Arachis hypogae and Neptunia sp. (Chandler 1978; Rivas et al. 2002). In the case of S. rostrata, which is an aquatic legume, an infection route through root hair curling by Azorhizobium caulinodans occurs under nonaquatic conditions, while infection by intercellular invasion occurs under flooded conditions. In addition to the infection modes, $S$. rostrata can form two types of nodules with $A$. caulinodans, determinate and indeterminate (Bomfeti et al. 2013; Ndoye et al. 1994). These two types of nodules can be differentiated by their morphology, physiology, and stage of development (Hirsch 1992). The plant hormone ethylene is a key factor controlling the type of nodules (Fernández-López et al. 1998). In addition to forming nodules with $S$. rostrata, A. caulinodans ORS571 could also infect many cereals such as rice and wheat (H. Liu et al. 2017).

The interaction between rhizobia and plants involves the chemotactic attraction of the bacteria toward the plant root system, followed by adsorption, colonization, proliferation, infection, and nodule morphogenesis. Chemotaxis is the first and essential process in the competitive rhizosphere environment (Scharf et al. 2016). In Escherichia coli, bacteria move toward the suitable niche via methyl-accepting chemoreceptors sensing extracellular signals. The presence of repellents or absence of attractants could increase the autokinase activity of CheA. CheA phosphorylates the response regulator $\mathrm{CheY}$, and the resulting phosphorylated CheY (CheY-P) regulates motor switching via binding to the flagellar motor protein FliM. CheY-P can be dephosphorylated by its own dephosphorylation ability. An additional phosphatase CheZ can accelerate the dephosphorylation 
of CheY-P and terminate the signal (Jones and Armitage 2015). For many bacteria such as Helicobacter pylori, Ralstonia solanacearum, Bacillus subtilis, and B. amyloliquefaciens, nonchemotactic mutants are significantly impaired in their colonization and invasion of the host roots (Aihara et al. 2014; Yao and Allen 2006; Yaryura et al. 2008) and, in particular, the initial colonization is impaired (Allard-Massicotte et al. 2016; McGee et al. 2005; Terry et al. 2005). In A. caulinodans, when the che gene cluster, including cheA, cheW, cheY2, cheB, and cheR, was deleted, the mutants were defective in competitive colonization and nodulation with host plants (W. Liu et al. 2018). In addition, two chemoreceptors encoded by $i c p B$ and $t$ lp $A l$ also were involved in the symbiosis in A. caulinodans (Jiang et al. 2016; W. Liu et al. 2017). Chemotaxis contributes to a competitiveness in the colonization process (Lacal et al. 2010; Vallad and Subbarao 2008). However, in contrast to what was observed for other chemotaxis mutants, early colonization was enhanced by A. caulinodans carrying a deletion of an orphan cheZ gene (X. Liu et al. 2018), and the mechanism of the unusual function of CheZ remains unknown.

Exopolysaccharides (EPS) play an important role in the establishment of an association between rhizobia and plants. EPS can protect rhizobia against plant defense compounds either as structural components or as signaling molecules (Mathis et al. 2005; Wakao et al. 2015). EPS are essential for the complete nodule development induced by Rhizobium leguminosarum CFN42 on legume plants (Cava et al. 1989). The modification of EPS can influence maize root colonization by Azospirillum brasilense (Jofré et al. 2004). In addition, a rhamnose-deficient EPS mutant of Rhizobium sp. IRBG74 is defective in symbiosis (Mitra et al. 2016). The symbiosis of Azorhizobium caulinodans with $S$. rostrata is also influenced by EPS production (Gao et al. 2001; Mathis et al. 2005). The EPS synthetized by A. caulinodans is composed of a linear homopolysaccharide made up of $\alpha$-1,3-linked 4,6-O-(1-carboxyethylidene)-D-galactosyl residues (D'Haeze et al. 2004). Two EPS biosynthesis-related gene clusters have been identified in the $A$. caulinodans genome (Tsukada et al. 2009). Cluster I is composed of four genes, including oac3 (azc_1831), oac2 (azc_1832), oac1 (azc_1833), and oac0 (azc_1834). Cluster II is composed of 14 genes, from $a z c \_3319$ to $a z c \_3332$ (Nakajima et al. 2012). The oac3 and oac2 genes encode a dTDP- ${ }_{-}-$glucose synthase and a dTDP $_{-}-$rhamnose synthase, respectively. The oac 3 and $o a c 2$ disrupted mutants failed to produce EPS and initiate the process of infection and thread formation (Gao et al. 2001; Goethals et al. 1994). However, previous reports are mostly focused on the effect of EPS on the later stages of the interaction between rhizobia and plants, and the role of EPS during early stages of the interaction remains unknown.

In this study, we assessed the role of the cheZ mutant in the nodulation process and in long-term colonization. In addition, we found that EPS production might be responsible for the competitive advantages of the cheZ mutant strain in the initial colonization compared with the wild type. This study may provide an insight to reconsider the roles of chemotaxis protein in host colonization.

\section{RESULTS}

\section{The enhanced competitive colonization}

of the cheZ mutant strain AC601 in the early stages.

We previously reported that CheZ in A. caulinodans negatively regulates the process of surface adhesion of bacterial cells on the root system (X. Liu et al. 2018). In the experimental design, previously used surface adhesion was determined after a relatively long time of incubation ( 4 and $24 \mathrm{~h}$ ) using inoculum of high cellular density (X. Liu et al. 2018). Therefore, to challenge former conclusions, a new set of experiments was performed using shorter incubation times and lower cell density.

Competitive colonization of the wild-type strain ORS571 and the cheZ mutant (strain AC601) with various inoculant
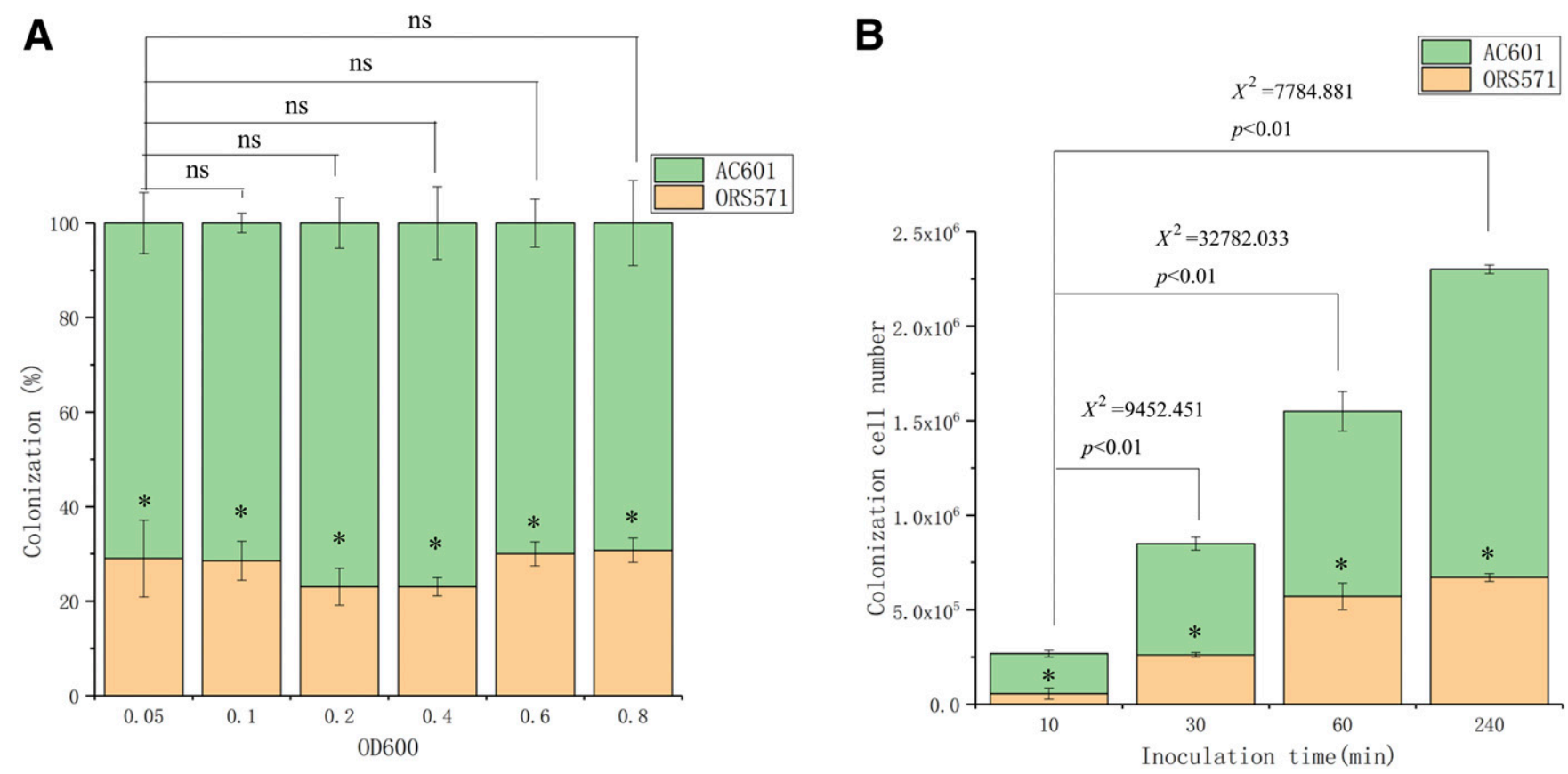

Fig. 1. Early-term competitive colonization of Sesbania rostrata seedlings by the Azorhizobium caulinodans wild-type and cheZ-deletion mutant strains. A, Dose response and $\mathbf{B}$, time response. Seedlings of $S$. rostrata were inoculated by wild-type strain ORS571 and the cheZ mutant strain AC601 mixed at equal concentrations. The relative colonization ratio of the wild type and the cheZ mutant was determined after $4 \mathrm{~h}$ in a dose response assay, and the initial concentration of bacteria was adjusted to an optical density at $600 \mathrm{~nm}$ of 0.6 in a time response assay. Data are means \pm standard deviation from at least three independent experiments. An asterisk $(*, P<0.01)$ indicates a Student's $t$ test of the mutant with respect to the wild type. The significant difference between different conditions is shown on the top of the column chart according to a $\chi^{2}$ analysis performed by SPSS; ns above the bars indicates not statistically different according to the $\chi^{2}$ analysis. 
concentrations of optical density at $600 \mathrm{~nm}\left(\mathrm{OD}_{600}\right)$ of 0.05 to 0.8 was analyzed after $240 \mathrm{~min}$ of incubation (Fig. 1A). Whatever the concentration of each strain, when the ratio between the wild type and the cheZ mutant was 1:1, quantification of the bacteria attached to the root system showed a higher number for the cheZ mutant than for the wild type, suggesting that the cheZ mutant had a competitive advantage at the early stages of the colonization process. Then, the experiment was repeated with an initial inoculation concentration of $\mathrm{OD}_{600}$ of 0.6 and quantification was performed and stopped at different times after inoculation (Fig. 1B). The number of bacterial cells on the roots increased with the inoculation time; however, the colonization ratios of the wild type and strain AC601 remained almost unchanged. During incubation, the number of strain AC601 mutants that adhered to the root surface of the host was approximately twofold greater than that of the wild type. These results showed that the competitive advantage of strain AC601 was robust and independent of population density and incubation time.

\section{The cheZ mutant strain AC601 was outcompeted in competitive nodulation but loss of cheZ could not affect effectiveness of nodules.}

Chemotaxis is an important factor in the process of competitive nodulation (Fig. 2). In order to investigate whether the enhanced colonization of the cheZ mutant could result in an increase of root nodulation, strains ORS571 and AC601 were inoculated at equal concentrations, and the proportion of their final nodule occupancy was analyzed. Because strain AC601 is defective in chemotaxis, the Sesbania seedlings were submerged into bacterial culture for $4 \mathrm{~h}(50 \mathrm{rpm})$ before being transplanted to Leonard jars filled with vermiculite. Surprisingly, in contrast to the results observed in early colonization experiments, the wild-type strain ORS571 had relatively higher nodule occupancy than strain AC601 in both competitive root nodulation and stem nodulation. To further confirm this result, we compared the relative competitive nodulation ability between the two strains from 10 days postinoculation (dpi) (when the nodules begin to be formed) to $43 \mathrm{dpi}$. The nodule number increased with time in strain ORS571; however, the occupancy of strain AC601 had no significant change, indicating that the nodulation ability of strain AC601 was limited under this stage. These results clearly showed that the enhanced colonization of strain AC601 could not result in an increase of the nodule occupancy.

In order to determine whether the defect of strain AC601 on competitive nodulation was caused by a defect in nitrogen fixation, we checked the effectiveness of nodules formed by strain AC601. Both strains ORS571 and AC601 can form root nodules that were pink in color (Supplementary Fig. S1). The pink color of nitrogen-fixing nodules results from the presence of oxygen-sequestering leghemoglobin (Barnett and Long 2018), indicating that the symbiotic properties are not influenced by CheZ. In addition, the wild type and cheZ mutant, when applied alone, had no significant difference on root nitrogen fixation abilities (Supplementary Fig. S2).

\section{Dynamics of long-term colonization of strain AC601 and the wild type.}

Because strain AC601 (cheZ mutant) could induce the development of normal nodules, we further determined whether the mutant strain had lost its competitive advantages during the long-term colonization process. The dynamic long-term colonization of strains ORS571 and AC601 on $S$. rostrata roots was monitored. First, strains ORS571 and AC601 were marked with LacZ (pXLGD4) and inoculated separately. Then, the distribution of strains ORS571 and AC601 on roots was tracked at different times. Interestingly, the colonization efficiency (degree of being dyed blue) of cheZ mutant strain AC601 was much more than that of the wild type after initial inoculation $(4 \mathrm{~h})$ whereas, at $3 \mathrm{dpi}$, the extent of colonization with strain AC601 or ORS571 was similar (Fig. 3). Over 5 days, the degree of strain ORS571 colonization was much more than that of strain AC601 and, near root tips, the difference between strains ORS571 and AC601 was more significant (Fig. 3).

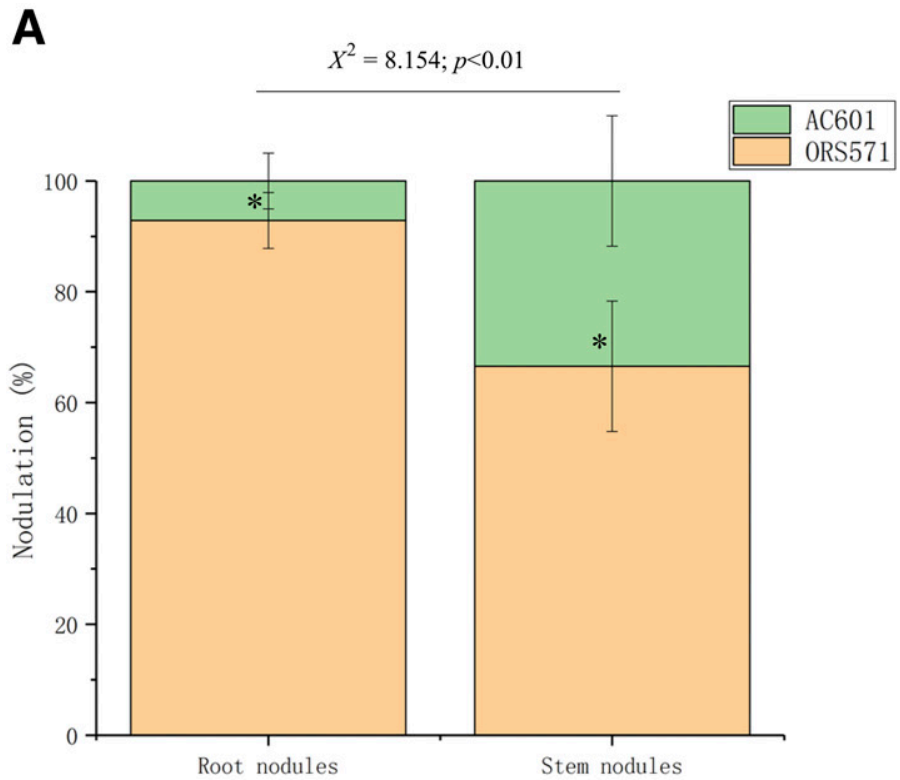

B
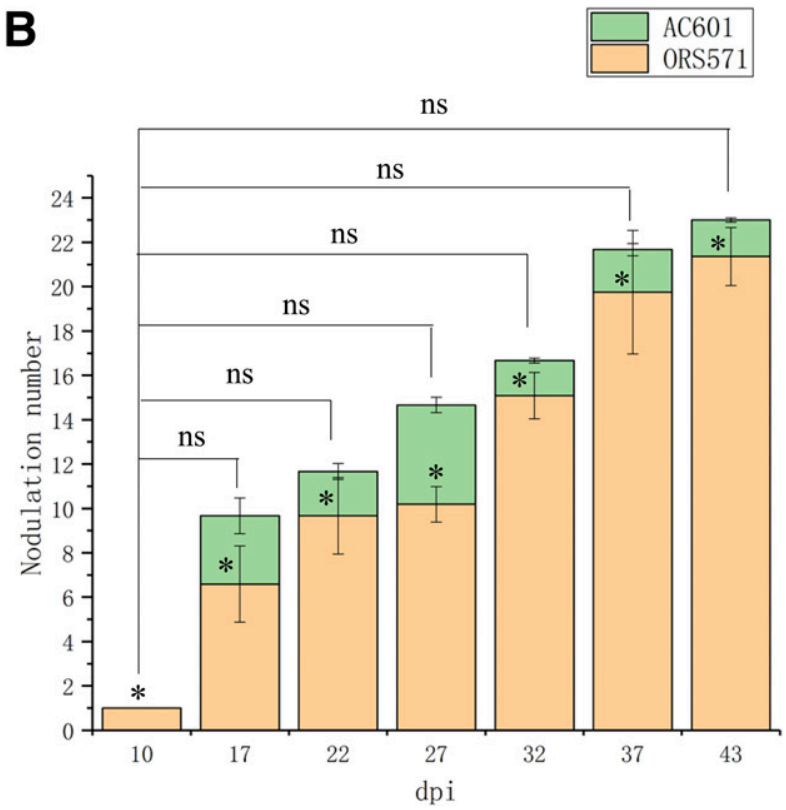

Fig. 2. Competitive nodulation of the wild type and the cheZ mutant. A, Root and shoot nodule occupancy of wild-type strain ORS571 and cheZ mutant strain AC601. B, Dynamics of root nodulation of the wild type and cheZ mutant; dpi $=$ days postinoculation. Values are means \pm standard deviation calculated from more than three independent experiments. An asterisk (*) indicates $P<0.01$ versus the wild type by a Student's $t$ test. A $\chi^{2}$ analysis was performed to show the significant difference between different conditions; ns above the bars indicates not statistically different according to the $\chi^{2}$ analysis. 
In addition, strains ORS571 and AC601 were mixed in equal proportion prior to inoculation, and their relative concentration in long-term competitive colonization (from $4 \mathrm{~h}$ to 15 days) along the root surface was analyzed (Fig. 4). In the root base region (0 to $2 \mathrm{~cm}$ ), strains AC601 and ORS571 were in equal ratio within 3 days, whereas strain AC601 was outcompeted by strain ORS571 after 5 days. In the fresh elongation region $(>3 \mathrm{~cm})$, strain ORS571 showed the most abundant cells
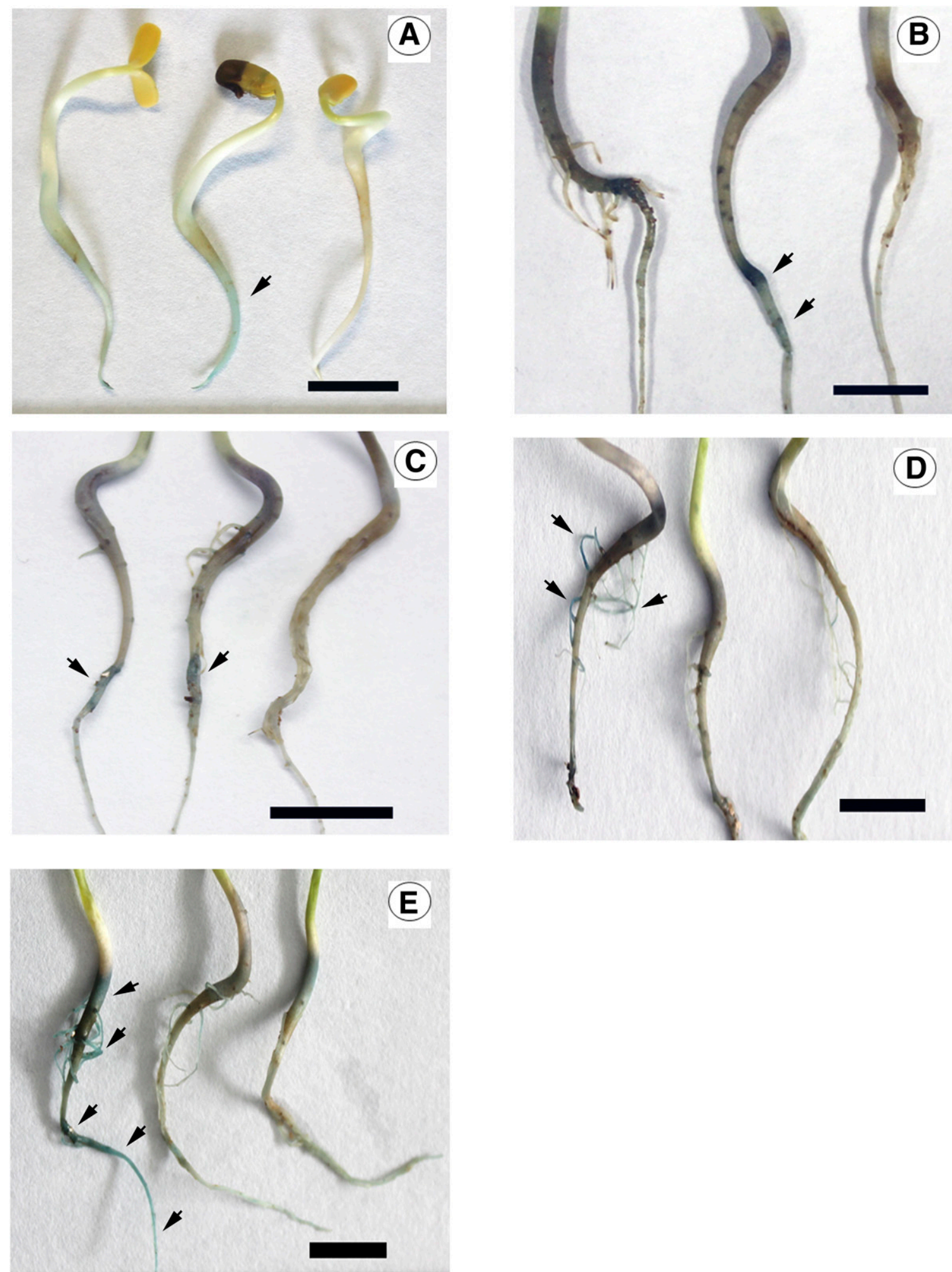

Fig. 3. Dynamics of long-term root surface colonization of wild-type strain ORS571 and cheZ mutant strain AC601. For each picture, seedlings inoculated with the wild type are positioned to the left, the cheZ mutant strain AC601 is in the middle, and the noninoculated control is on the right. Inoculated strains carry pXLGD4 containing the lacZ gene. In situ 5-bromo-4-chloro-3-indolylbeta-D-galacto-pyranoside staining was performed after A, $4 \mathrm{~h}$ postinoculation; $\mathbf{B}, 3$ days postinoculation (dpi); C, $5 \mathrm{dpi} ; \mathbf{D}, 7 \mathrm{dpi}$; and $\mathbf{E}, 10 \mathrm{dpi}$. Black arrows indicate the sites on the plant with significant difference between plants inoculated with the wild type and the cheZ mutant. 
colonizing the root surface, and strain AC601 showed gradually decreased occupancy alongside the root surface in the direction of root tip. The number of nodules of the wild type was approximately 60-fold more than that of the cheZ mutant in the root region near the tip ( 4 to $5 \mathrm{~cm}$ ) at $10 \mathrm{dpi}$ whereas, at the base of the roots, the number of nodules of the wild type was approximately 2.5-fold higher than that of cheZ mutant. These results suggested that the competitive advantages of the cheZ mutant in early colonization are lost in long-term colonization, and CheZ helps A. caulinodans spread alongside the root surface.

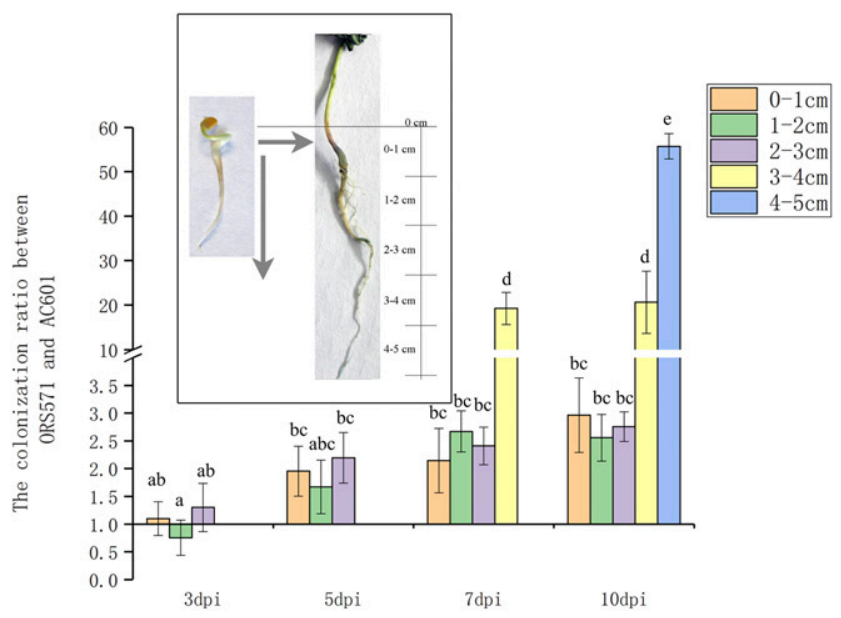

Fig. 4. Dynamics of competitive long-term colonization of the wild type and the cheZ mutant. In the dynamic of competitive long-term colonization, wild-type strain ORS571 and cheZ mutant strain AC601 were inoculated in equal proportions. The distance from the site of the nodule to the base of the root is indicated in centimeters; dpi = days postinoculation. The insert shows the difference in growth of Sesbania plantlets from 1 to 11 days. The distance from the base of the root is marked with the scale. Data are expressed as means \pm standard deviation calculated from three independent experiments. The same letter above the error bars indicates not statistically different according to Duncan's test $(P<0.05)$.
The distribution of root nodules formed by strains ORS571 and AC601.

The inversion in long-term colonization led us to further investigate whether the decrease of the cheZ mutant in competitive nodulation results from the decrease in long-term colonization. The distribution of nodules formed by strains ORS571 and AC601 along root surface was characterized, including the number and relative location of nodules. When strains ORS571 and AC601 were inoculated separately, the total number of nodules formed by strains ORS571 and AC601 was similar (Fig. 5A). However, the distribution frequencies of nodules at different positions were significantly different between strains ORS571 and AC601. Most nodules formed by strain AC601 were only found in the root base region, while many nodules of the wild type were located in the root elongation region, toward the root tip (Fig. 5B). These results showed that Che $\mathrm{Z}$ contributes to the distribution of nodules.

Then, the distribution of nodules formed by strains ORS571 and AC601 in competitive nodulation assay was determined. When strain ORS571 was mixed with strain AC601 at a 1:1 ratio, the highest numbers of nodules were found at the root base, whereas numbers of nodules gradually decreased in the direction of the root tip (Supplementary Fig. S3). Interestingly, all the nodules formed by strain AC601 were located in the initial susceptible region, and numbers of nodules formed by strain AC601 were less than that formed by strain ORS571 in this region. These results are consistent with the results when strain AC601 and the wild type inoculated separately in longterm colonization, indicating that the che $\mathrm{Z}$ mutant could not disperse freely.

\section{EPS may be the key factor contributing}

to the enhanced early colonization of the cheZ mutant.

Similar to the cheZ mutant, nonchemotaxis mutants in Sinorhizobium meliloti can form normal nodules but had relatively low nodule occupancy in competitive nodulation with the wild type (Caetano-Anollés et al. 1988). In R. leguminosarum bv. trifolii, production of EPS significantly impaired bacterial attachment and biofilm formation on biotic surfaces (Janczarek et al. 2015). In

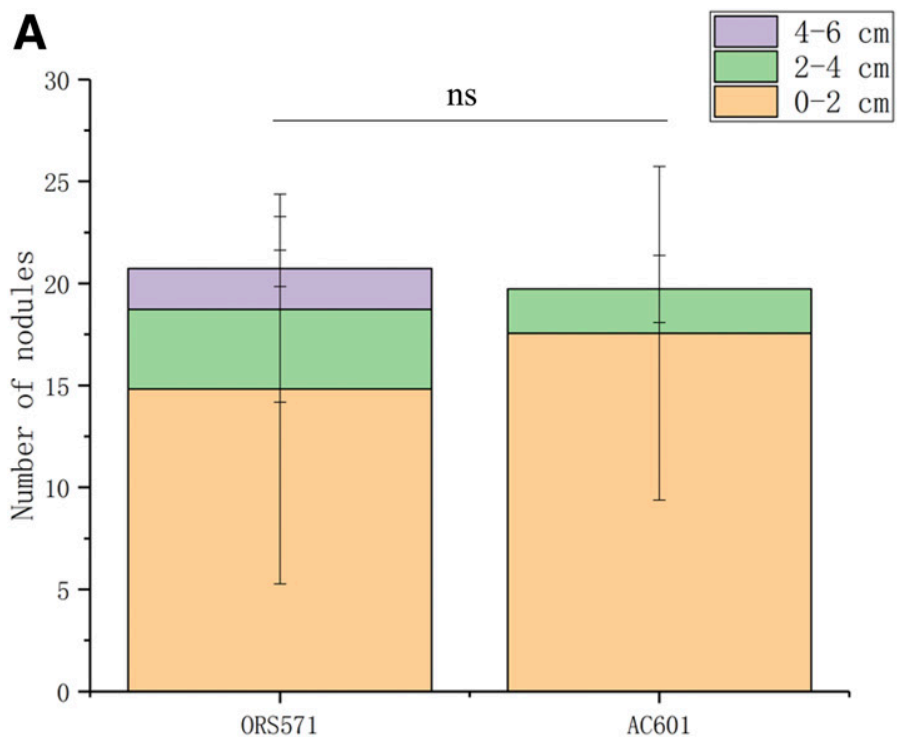

B

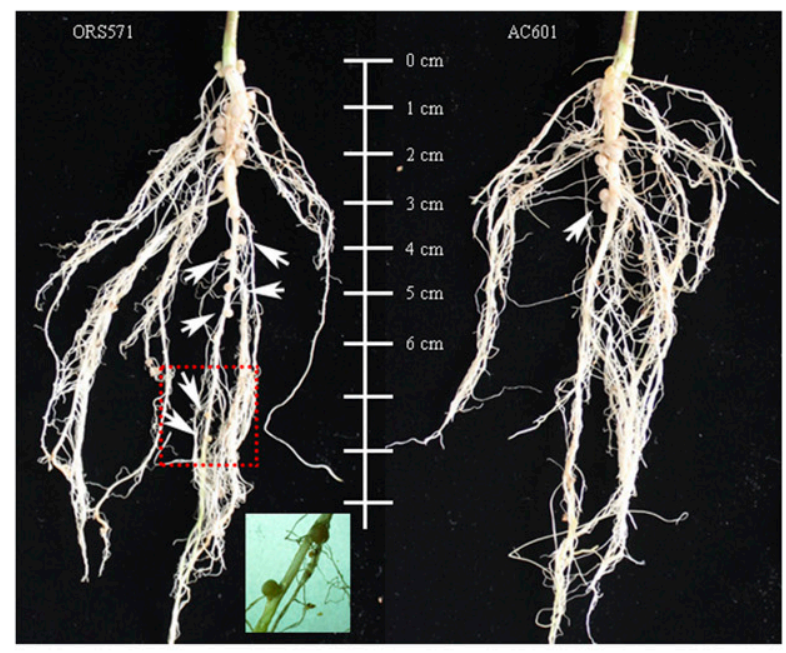

Fig. 5. Difference in the distribution of the root nodules formed on Sesbania plantlets inoculated by Azorhizobium caulinodans wild-type strain ORS571 or by the cheZ mutant strain AC601. A, Diagrammatic representation of the number of nodules formed with respect to their distance from the root base: between 0 and $2 \mathrm{~cm}$ in yellow, 2 and $4 \mathrm{~cm}$ in green, and 4 and $6 \mathrm{~cm}$ in violet. B, Root picture showing nodules localization: the white arrow points to the position of nodules away from the root base. The farthest and youngest nodules in Sesbania plantlets by the wild type were magnified. Error bars show \pm standard deviation of the means from at least three independent experiments; ns at the top of the column chart indicate no significant difference between the mutant and wild-type strains according to a $\chi^{2}$ analysis performed by SPSS. 
addition, EPS molecules could promote the formation of infection threads of $R$. phaseoli (Carlson et al. 1987). In order to investigate the role of EPS in the early-term colonization of the cheZ mutant, the expression levels of two gene clusters encoding EPS biosynthesis proteins were analyzed (Fig. 6A and B). The relative expression levels of genes in the two clusters all increased more than twofold in the cheZ mutant compared with the wild type, especially oac3 (azc_1831). These results indicated that EPS are involved in the CheZ-regulated colonization.

To further investigate the contribution of EPS in the colonization process, a strain carrying a deletion of the oac 3 gene (strain AC1831) and a double mutant strain carrying deletions of both oac3 and cheZ (strain AC6031) were constructed. The Congo red assay and anthrone method were used to assess the production of EPS produced by these strains qualitatively and quantitatively. EPS production by the cheZ mutant (strain AC601) was much higher than that of the wild type, which was in agreement with our previous findings (X. Liu et al. 2018).

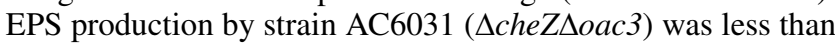
that of strain AC601 ( $\triangle c h e Z$ ) (Fig. 6C), and strain AC6031 showed a significant decrease in a competitive colonization assay with strain ORS571 (wild type), which was similar to strain AC1831 (Doac3) (Fig. 6D), indicating that the increase of EPS production may play important roles in early colonization.

It has been reported that the EPS production is more critical for symbiosis on plant hosts that form indeterminate nodules than on those that form determinate nodules (Mendis et al. 2016). In order to determine whether the type of nodules was affected
A

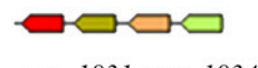

azc 1831-azc 1834

B

$B$
0
0
0
0
0
0
0
0
0
0
0
0
0
0
0
0

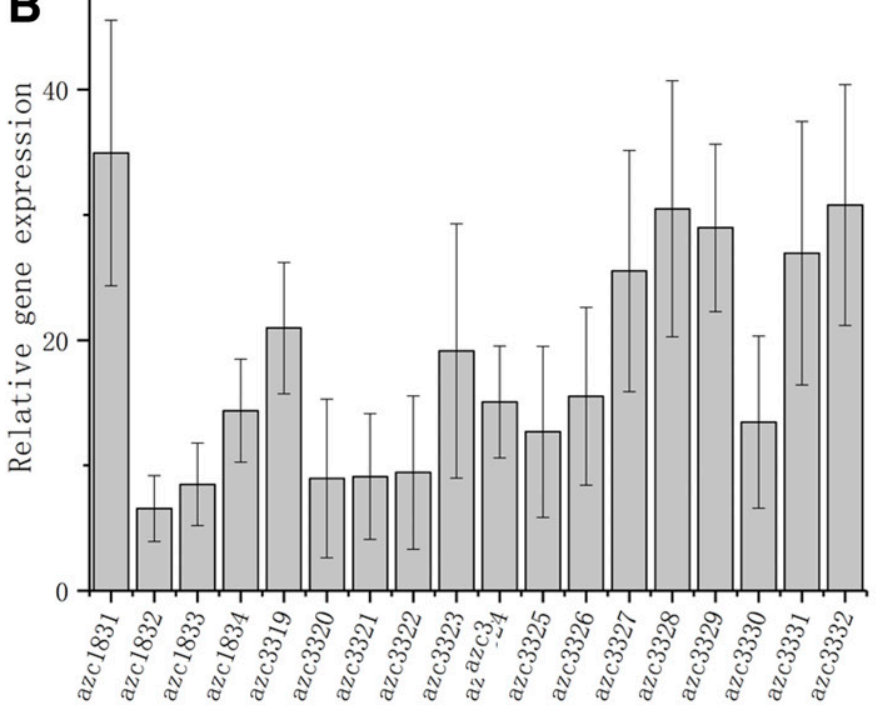

C

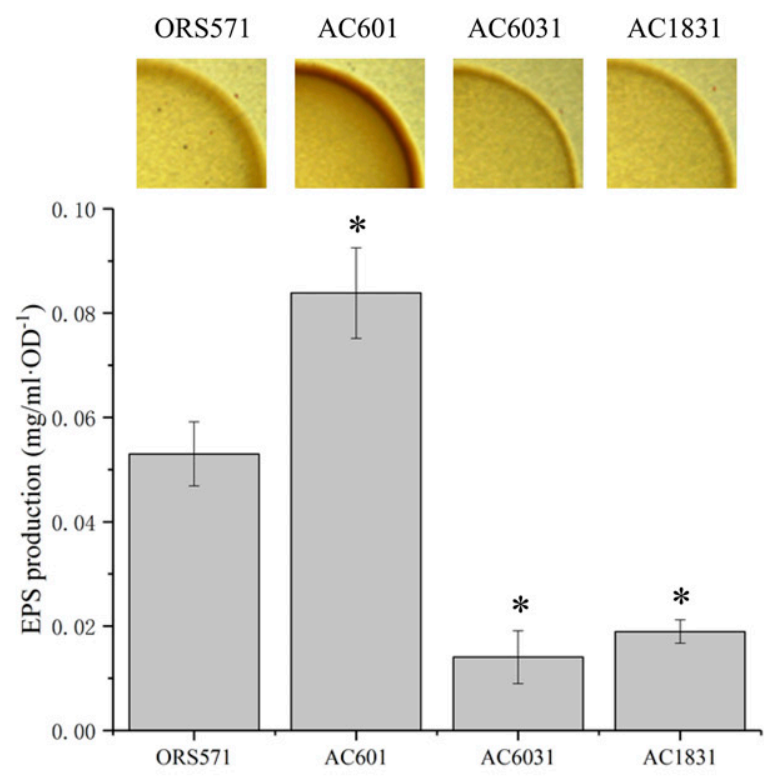

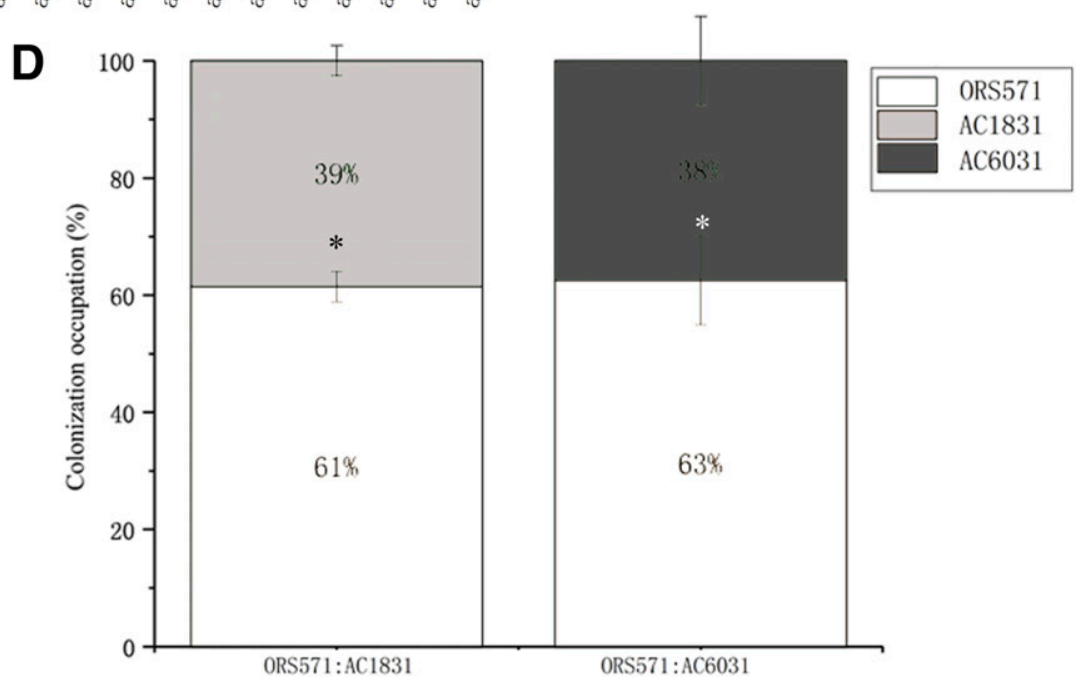

Fig. 6. Relative expression of Azorhizobium caulinodans exp genes in the cheZ mutant strain AC601 compared with the wild type. A, Schematic representation of the two exp clusters in the A. caulinodans genome. Cluster I is composed of $a z c \_1831$ (oac3), azc_1832,azc_1833, and azc_1834. Cluster II is composed of genes from $a z c_{\_} 3319$ to $a z c_{-} 3332$. B, Relative expression of all 17 exp genes increased more than fivefold in the $c h e Z$ mutant compared with the wild type. C, Qualitative and quantitative production of exopolysaccharides (EPS) revealed by Congo red staining (top) or by determination in the culture supernatant using the assay described in text (bottom). Strains: ORS571 = wild type, AC601 = cheZ mutant, AC1831 = oac3 mutant, and AC6031 = cheZoac3 double mutant. White colonies of AC1831 and AC6031 indicated the lack of EPS production. D, Competitive colonization of different strains. Values are means of a standard deviation from at least three independent experiments. Asterisks $\left(^{*}\right)$ indicate a significant difference between the wild type and each mutant according to a Student's $t$ test. 
by CheZ, the number of nodules of different types was counted when inoculated with strain AC601 ( $\Delta c h e Z)$ or strain ORS571 (wild type). However, there was no significant difference in the type of nodules between them (Supplementary Fig. S4).

\section{DISCUSSION}

The rhizosphere is a complex environment, and it was established that chemotaxis is important for bacteria to construct effective association with plants (Miller et al. 2007). In $R$. solanacearum, a nonchemotactic mutant could not locate and infect the host plant effectively (Yao and Allen 2006). B. subtilis needs chemotaxis to swim toward Arabidopsis thaliana roots (Allard-Massicotte et al. 2016). We found that an essential chemotactic gene, cheZ, has a negative effect on earlyterm microbe-plant interaction, which is in contrast to the effect of other chemotactic gene deletions in Azorhizobium caulinodans (W. Liu et al. 2018).

When inoculated alone, numbers and nitrogen fixation activities of the nodules induced by the cheZ mutant are similar to those of the wild type. Although chemotaxis and motility are not necessary for infection and nodulation, they could confer a significant advantage during these processes (Caetano-Anollés et al. 1988). There is a strong relationship between attachment to the plant root surface and final successful nodulation for rhizobacteria (de Weert et al. 2004). In R. meliloti and Bradyrhizobium japonicum, motility and chemotaxis significantly increased the initial contact and adsorption of rhizobia to the host root surface, leading to an increase of the efficiency of nodulation (Althabegoiti et al. 2008; Caetano-Anollés et al. 1988). However, when coinoculated with the wild type and the cheZ mutant, the nodule occupancy of the cheZ mutant was lower than that of the wild type. It is obvious that the competitive advantages of the cheZ mutant in early colonization cannot result in the increase of nodule number. Through tracking the dynamics of long-term colonization of the cheZ mutant and the wild type separately, we found that the colonization level of the cheZ mutant was less than that of the wild type after 5 days. When coinoculated with the wild type, the relative colonization level of the cheZ mutant near the root tip decreased faster, indicating that chemotaxis is important in a competitive environment. It is suggested that CheZ plays an essential role in long-term competitive colonization and, once che $Z$ is disrupted, the strains cannot successfully establish effective association with host plants in a complex environment.

In general, nonchemotactic mutants are defective in initial host colonization, yet the cheZ mutant strain shows an unusual phenotype in early colonization. What is the reason for the competitive advantage of the cheZ mutant? There are two possible explanations implying (i) the alternative cellular function (ACF) (different from motility) associated with the chemotaxis pathway or (ii) the swimming motility in relation to EPS production. (i) For bacteria containing several chemotaxis systems, only one (major) chemotaxis system is involved in the regulation of motility and chemotaxis, while the second or other chemotaxis systems are responsible for ACF such as biofilm formation and EPS production (Black and Yang 2004; Hickman et al. 2005). For other bacteria, chemotaxis and ACF such as adhesion and biofilm can be performed by the same chemotaxis system. For example, the deletion of cheA or cheB in Marinobacter adhaerens resulted in impairment on swimming and biofilm formation at the same time (Sonnenschein et al. 2012). Recently, cross talk was found between the major and minor chemosensory systems on biofilm formation in Comamonas testosteroni CNB-1 (Huang et al. 2019). Our group has found that the chemotaxis, flocculation, biofilm formation, and EPS production were regulated by one chemotaxis system in A. caulinodans ORS571, and that the roles of CheA and CheZ antagonize each other during these processes (W. Liu et al. 2018; X. Liu et al. 2018). Deletion of cheZ led to improvement of both biofilm formation and EPS production (X. Liu et al. 2018). The inhibition of CheZ on EPS production might be a critical factor for early-term colonization of $A$. caulinodans ORS571. (ii) The altered swimming mode of $c h e Z$ mutant affects bacterial interactions with surfaces. For many $\alpha$-Proteobacteria such as Asticcacaulis biprosthecum, Caulobacter crescentus, and Agrobacterium tumefaciens, the contact of cells with a surface could cause an increased synthesis of EPS and promote adhesion to the surface (Belas 2014). The hyperreversal behavior of the cheZ mutant may enhance the strength of flagella hitting a solid surface and induce the regulation of mechanosensing on EPS production.

CheZ did not affect nodule formation (number) when inoculated alone; however, the cheZ mutant showed a reduced nodule occupancy in a competitive nodulation assay, and the distribution and location of nodules were significantly different between the cheZ mutant and the wild type. Nodules can be formed alongside the main root surface of Sesbania spp. When the wild type or the cheZ mutant was inoculated solely, most nodules were developed in the initial colonizing region of roots. However, nearly no bacterial cells in the cheZ mutant can move away from the initial inoculation region $(0$ to $4 \mathrm{~cm})$. In the competitive nodulation assay, the colonization region of cheZ mutant was even narrower ( 0 to $2 \mathrm{~cm})$, indicating that CheZ is important for the dispersal of nodules along the root. What is the reason for the decreased ability of the cheZ mutant to form nodules along young plant roots? (i) The limited areas of colonization and nodulation of the cheZ mutant may be consistent with the role of CheZ on swimming ability. Because the the wild type displays high efficiency in spreading and utilizing limiting nutrients on the root surface, it is unsurprising that the colonization regions of the cheZ mutant become even narrower when coinoculated with the wild type. (ii) EPS, on the one hand, can form a diffusion barrier to protect Azorhizobium caulinodans against $\mathrm{H}_{2} \mathrm{O}_{2}$ (D'Haeze et al. 2004). On the other hand, EPS overproduction might inhibit the migration of the cheZ mutant. When we had previously observed the trajectory of the cheZ mutant, we found that many cells adhered to the surface of slides, although they were shaking violently and trying to break free (X. Liu et al. 2018). (iii) The cheZ mutant is less competitive during infection. At the initial site of colonization, the advantage of the cheZ mutant on early colonization can be overturned by the wild type during long-term symbiosis, indicating that nonchemotactic mutants are defective in the maintenance of infection (Terry et al. 2005).

EPS are important for attachment to various surfaces, including root surface (Ma et al. 2006). Overproduction of EPS causes significantly increased adhesion of Pseudomonas aeruginosa (Ma et al. 2006), and EPS are also required for the attachment of Azospirillum brasilense (Jofré et al. 2004). When the EPS biosynthesis genes are disrupted in Rhizobium sp. IRBG74, the resulting mutant strains are defective not only in colonization but also in nodulation (Mitra et al. 2016). In Azorhizobium caulinodans ORS571, the roles of EPS in the formation of infection threads and nodulation have been studied (Mitra et al. 2016; Wakao et al. 2015). Consistent with previous reports (Gao et al. 2001, Goethals et al. 1994), deletion of oac3 abolished the EPS production in A. caulinodans ORS571. In the present work, a mutant strain carrying a deletion of both oac3 and cheZ (strain AC6031) not only resulted in a decrease of EPS production but also showed reduced early colonization, a phenotype which is similar to that of the oac3 mutant strain (AC1801) but different from the cheZ mutant (strain AC601), indicating that the enhanced early-term colonization of the cheZ mutant might depend on the increase of EPS production. 
In addition, the enhanced expression of eps synthesis genes in the cheZ mutant further indicated that EPS may be the key factor in cheZ mutant early- and long-term colonization.

As quoted before, multiple chemosensory systems in bacteria control ACF. They are involved in the control of EPS production (Black and Yang 2004), biofilm formation (Huangyutitham et al. 2013), motility by type IV pili (Bhaya et al. 2001), flagella biosynthesis (Berleman and Bauer 2005a), fruiting-body formation (Willett and Kirby 2011), developmental gene expression regulation (Kirby and Zusman 2003), cyst formation (Berleman and Bauer 2005b), and swarm cell differentiation (Burkart et al. 1998). For example, in Myxococcus xanthus, the EPS production is regulated by the Dif chemosensory system, which could not regulate motility (Black et al. 2015). Recently, in C crescentus, chemotactic protein CheB, encoded by a cheB gene that is not part of the major chemotaxis operon, was found to play a role in surface attachment, antagonizing the role of CheA (Berne and Brun 2019). Although CheZ in A. caulinodans also functions in an antagonistic manner with CheA, it could regulate EPS production and chemotactic motility at the same time.

It has been reported that flagella-mediated chemotaxis enables bacteria to reach suitable niches but is not essential for the subsequent contacts of bacteria with the host plants (Coskun et al. 2017). In this work, we found that CheZ cannot only regulate early attachment via affecting EPS production but also influences the dispersal of bacteria along root surface in longterm colonization. The effect of chemotactic proteins in plant-microbe relationships at the colonization level could enable us to use microbes to enhance agriculture productivity effectively.

\section{MATERIALS AND METHODS}

\section{Media and growth conditions.}

The bacterial strains and plasmids used in this study are listed in Table 1. The A. caulinodans strains (Dreyfus et al. 1988), including the wild type and its derivatives, were cultured at $37^{\circ} \mathrm{C}$ in tryptone-yeast extract (TY) medium overnight. A. caulinodans strains were added with antibiotics at the following final concentrations: ampicillin at $100 \mu \mathrm{g} / \mathrm{ml}$; nalidixic acid at $25 \mu \mathrm{g} / \mathrm{ml}$; gentamicin at $50 \mu \mathrm{g} / \mathrm{ml}$; kanamycin at $50 \mu \mathrm{g} / \mathrm{ml}$; and tetracycline at $100 \mu \mathrm{g} / \mathrm{ml}$.

\section{Plasmid and strain construction.}

To construct the oac3 mutant, a 626-bp upstream fragment and a 751-bp downstream fragment of oac3 were amplified by PCR. The amplicons of the upstream fragment and allelic exchange vector pCM351 (Marx and Lidstrom 2002) were digested with restriction enzymes $(K p n \mathrm{I}$ and $N d e \mathrm{I})$; then, they were linked with $\mathrm{T} 4$ ligase. The resulting construct and downstream fragment were digested with restriction enzymes (AgeI and SacI) and linked by T4 ligase. The modified pCM351 containing the upstream and downstream fragments of oac3 was introduced into strain ORS571 by triparental conjugation for allelic exchange with the helper pRK2013, as previously described (Ditta et al. 1980). Homologous recombinants carrying a deletion of the $o a c 3$ gene were selected on TY plates containing gentamicin, nalidixic acid, and ampicillin. It was checked that the recombinant strain cannot grow on plates containing tetracycline. Double recombination was confirmed by PCR and sequencing. The selected strain, named AC1831, was used in subsequent experiments.

The cheZ mutant strain AC601 was used to obtain a doublemutant strain carrying a deletion of oac 3 and cheZ genes. First, the gentamicin gene was removed using pCM157, as previously described (Marx and Lidstrom 2002). Then, the resulting unmarked cheZ mutant was used to construct a double-deletion

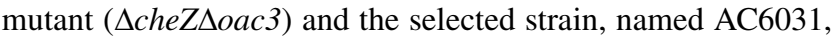
was used in subsequent experiments.

\section{Plant growth.}

Seed sterilization and germination were performed as previously described (Jiang et al. 2016). Seedlings of similar root size were selected and used in subsequent experiments.

\section{Early-term colonization assay.}

For early-term colonization, strains ORS571 and AC601 were mixed with equal ratios and plants were soaked in the cell suspension. Incubation was performed with shaking at $50 \mathrm{rpm}$, at the time course indicated in the Results section. The bacteria tightly attached to the roots were collected and quantified. Adsorbed rhizobia were collected from the root surfaces, and the recovered isolates from surfaces were identified by PCR (Mongiardini et al. 2008). During the time-course assay, Sesbania roots were inoculated from 10 to $240 \mathrm{~min}$.

\section{Long-term colonization assay.}

Seedlings were soaked in bacterial suspensions using A. caulinodans strains marked with lacZ. The concentration of bacteria was adjusted to an $\mathrm{OD}_{600}$ of 0.6. Then, roots were stained with 5-bromo-4-chloro-3-indolylbeta-D-galacto-pyranoside and the colonization patterns at different times were analyzed, as previously reported (Qiu et al. 2015). For competitive long-term colonization assay, strains ORS571 and AC601 were mixed at equal proportions prior to inoculation. After growing for different periods of time, roots were washed at least five times, and the different regions of roots were collected separately. The roots were vortexed and bacteria were reisolated on TY solid agar plates containing nalidixic acid and ampicillin. The proportion between strains ORS571 and AC601 was quantified by TY solid plates containing gentamicin, where strain ORS571 could not survive.

Table 1. Bacteria strains and plasmids used in this study

\begin{tabular}{|c|c|c|}
\hline Strain or plasmid & Relevant characteristics $^{\mathbf{a}}$ & Source or reference \\
\hline \multicolumn{3}{|l|}{ Strains } \\
\hline \multicolumn{3}{|l|}{ Escherichia coli } \\
\hline DH5 $\alpha$ & $\mathrm{F}^{-}$supE44 AlacU169 ( $\phi 80$ lacZLM15) hsdR17 recA1 endA1 gyrA96 thi-1 relA1 & Transgen \\
\hline \multicolumn{3}{|c|}{ Azorhizobium caulinodans } \\
\hline ORS571 & Type strain; $\mathrm{Am}^{\mathrm{R}}, \mathrm{Na}^{\mathrm{R}}$ & Dreyfus et al. 1988 \\
\hline AC601 & ORS571 derivative; $\Delta c h e Z, \mathrm{Am}^{\mathrm{R}}, \mathrm{Na}^{\mathrm{R}}, \mathrm{Gm}^{\mathrm{R}}$ & X. Liu et al. 2018 \\
\hline AC1831 & ORS571 derivative, $\Delta o a c 3, \mathrm{Am}^{\mathrm{R}}, \mathrm{Na}^{\mathrm{R}}, \mathrm{Gm}^{\mathrm{R}}$ & This study \\
\hline AC6031 & AC601 derivative, $\Delta$ cheZ and $\Delta o a c 3, \mathrm{Am}^{\mathrm{R}}, \mathrm{Na}^{\mathrm{R}}, \mathrm{Gm}^{\mathrm{R}}$ & This study \\
\hline \multicolumn{3}{|c|}{$\mathrm{cos}$} \\
\hline pCM351 & Allelic exchange vector, $\mathrm{Gm}^{\mathrm{R}}, \mathrm{Tc}^{\mathrm{R}}$ & Marx and Lidstrom 2002 \\
\hline pCM157 & Allelic exchange vector, $\mathrm{Tc}^{\mathrm{R}}$ & Marx and Lidstrom 2002 \\
\hline pRK2013 & Helper plasmid, ColE1 replicon; $\operatorname{Tr}^{\mathrm{R}}, \mathrm{Km}^{\mathrm{R}}$ & Ditta et al. 1980 \\
\hline
\end{tabular}

${ }^{a} \mathrm{Am}^{\mathrm{R}}, \mathrm{Na}^{\mathrm{R}}, \mathrm{Gm}^{\mathrm{R}}, \mathrm{Tc}^{\mathrm{R}}$, and $\mathrm{Km}^{\mathrm{R}}=$ resistant to ampicillin, nalidixic acid, gentamycin, tetracycline, and kanamycin, respectively. 


\section{Nodulation of Sesbania rostrata.}

For root nodulation assays, surface-sterilized seedlings that were similar in size and length were selected and inoculated with A. caulinodans strains adjusted to an $\mathrm{OD}_{600}$ of 0.6 for $4 \mathrm{~h}$. Then, seedlings were transplanted into Leonard jars filled with sterilized vermiculite. The nodulation pattern was analyzed after approximately 45 days. For stem nodulation assays, after Sesbania rostrata grew for 4 weeks, A. caulinodans strains adjusted to an $\mathrm{OD}_{600}$ of 0.6 were uniformly painted alongside the stem surface. The stem nodulation pattern was analyzed after another 4 weeks.

\section{Expression analysis of EPS biosynthesis-related genes.}

Strains ORS571 and AC601 were cultured in TY liquid medium at $37^{\circ} \mathrm{C}$ for $24 \mathrm{~h}$. TransZol Up Plus RNA kit (Transgen Biotech) was used to isolate total RNA from $25 \mathrm{ml}$ of A. caulinodans cells. After determining the concentration and quality of RNA via Nanodrop 2000, a One-Step gDNA removal kit (Transgen Biotech) was used to synthesize cDNA for subsequent experiments. GoTaq qPCR Master Mix kit (Promega Corp.) was used to perform real-time quantitative PCR with the gene-specific primer pairs listed in Supplementary Table S1. The transcript levels of 16sRNA were used as a control, and the comparative cycle threshold method (Schmittgen and Livak 2008) was used to analyze the transcript levels of $\exp$ genes.

\section{Qualitative and quantitative assays of EPS production.}

The qualitative assay of EPS production was performed as previously described (Nakajima et al. 2012), with some modifications, as described by Jiang et al. (2016). Qualitative determination using Congo red staining was as described by Jiang et al. (2016). The quantitative assay of EPS production was performed using the anthrone method with some modifications, as described by X. Liu et al. (2018). These experiments were repeated at least three times, with six replicates per sample.

\section{ACKNOWLEDGMENTS}

We thank C. Elmerich for helpful and insightful comments on the manuscript and F. Xie for providing vector pXLGD4.

\section{LITERATURE CITED}

Aihara, E., Closson, C., Matthis, A. L., Schumacher, M. A., Engevik, A. C., Zavros, Y., Ottemann, K. M., and Montrose, M. H. 2014. Motility and chemotaxis mediate the preferential colonization of gastric injury sites by Helicobacter pylori. PLoS Pathog. 10:e1004275.

Allard-Massicotte, R., Tessier, L., Lécuyer, F., Lakshmanan, V., Lucier, J. F., Garneau, D., Caudwell, L., Vlamakis, H., Bais, H. P., and Beauregard, P. B. 2016. Bacillus subtilis early colonization of Arabidopsis thaliana roots involves multiple chemotaxis receptors. MBio 7:e01664-16.

Althabegoiti, M. J., López-García, S. L., Piccinetti, C., Mongiardini, E. J., Pérez-Giménez, J., Quelas, J. I., Perticari, A., and Lodeiro, A. R. 2008. Strain selection for improvement of Bradyrhizobium japonicum competitiveness for nodulation of soybean. FEMS Microbiol. Lett. 282:115-123.

Barnett, M. J., and Long, S. R. 2018. Novel genes and regulators that influence production of cell surface exopolysaccharides in Sinorhizobium meliloti. J. Bacteriol. 200:e00501-17.

Belas, R. 2014. Biofilms, flagella, and mechanosensing of surfaces by bacteria. Trends Microbiol. 22:517-527.

Berleman, J. E., and Bauer, C. E. 2005a. A che-like signal transduction cascade involved in controlling flagella biosynthesis in Rhodospirillum centenum. Mol. Microbiol. 55:1390-1402.

Berleman, J. E., and Bauer, C. E. 2005b. Involvement of a Che-like signal transduction cascade in regulating cyst cell development in Rhodospirillum centenum. Mol. Microbiol. 56:1457-1466.

Berne, C., and Brun, Y. 2019. The two chemotaxis in Caulobacter crescentus operons play different roles in chemotaxis and biofilm regulation. bioRxiv . doi:10.1101/528224
Bhaya, D., Takahashi, A., and Grossman, A. R. 2001. Light regulation of type IV pilus-dependent motility by chemosensor-like elements in Synechocystis PCC6803. Proc. Natl. Acad. Sci. U.S.A. 98:7540-7545.

Black, W. P., Wang, L., Davis, M. Y., and Yang, Z. 2015. The orphan response regulator EpsW is a substrate of the DifE kinase and it regulates exopolysaccharide in Myxococcus xanthus. Sci. Rep. 5: Article 17831.

Black, W. P., and Yang, Z. 2004. Myxococcus xanthus chemotaxis homologs DifD and DifG negatively regulate fibril polysaccharide production. J. Bacteriol. 186:1001-1008.

Bomfeti, C. A., Ferreira, P. A., Carvalho, T. S., De Rycke, R., Moreira, F. M., Goormachtig, S., and Holsters, M. 2013. Nodule development on the tropical legume Sesbania virgata under flooded and non-flooded conditions. Plant Biol. (Stuttg.) 15:93-98.

Burkart, M., Toguchi, A., and Harshey, R. M. 1998. The chemotaxis system, but not chemotaxis, is essential for swarming motility in Escherichia coli. Proc. Natl. Acad. Sci. U.S.A. 95:2568-2573.

Caetano-Anollés, G., Wall, L. G., De Micheli, A. T., Macchi, E. M., Bauer, W. D., and Favelukes, G. 1988. Role of motility and chemotaxis in efficiency of nodulation by Rhizobium meliloti. Plant Physiol. 86:1228-1235.

Carlson, R. W., Kalembasa, S., Turowski, D., Pachori, P., and Noel, K. D. 1987. Characterization of the lipopolysaccharide from a Rhizobium phaseoli mutant that is defective in infection thread development. J. Bacteriol. 169:4923-4928.

Cava, J. R., Elias, P. M., Turowski, D. A., and Noel, K. D. 1989. Rhizobium leguminosarum CFN42 genetic regions encoding lipopolysaccharide structures essential for complete nodule development on bean plants. J. Bacteriol. 171:8-15.

Chandler, M. R. 1978. Some observations on infection of Arachis hypogaea L. by rhizobium. J. Exp. Bot. 29:749-755.

Coskun, D., Britto, D. T., Shi, W., and Kronzucker, H. J. 2017. How plant root exudates shape the nitrogen cycle. Trends Plant Sci. 22:661-673.

de Weert, S., Kuiper, I., Lagendijk, E. L., Lamers, G. E., and Lugtenberg, B. J. 2004. Role of chemotaxis toward fusaric acid in colonization of hyphae of Fusarium oxysporum f. sp. radicis-lycopersici by Pseudomonas fluorescens WCS365. Mol. Plant-Microbe Interact. 17:1185-1191.

D'Haeze, W., Glushka, J., De Rycke, R., Holsters, M., and Carlson, R. W. 2004. Structural characterization of extracellular polysaccharides of Azorhizobium caulinodans and importance for nodule initiation on Sesbania rostrata. Mol. Microbiol. 52:485-500.

Ditta, G., Stanfield, S., Corbin, D., and Helinski, D. R. 1980. Broad host range DNA cloning system for gram-negative bacteria: Construction of a gene bank of Rhizobium meliloti. Proc. Natl. Acad. Sci. U.S.A. 77:7347-7351.

Dreyfus, B., Garcia, J.-L., and Gillis, M. 1988. Characterization of Azorhizobium caulinodans gen. nov., sp. nov., a stem-nodulating nitrogen-fixing bacterium isolated from Sesbania rostrata. Int. J. Syst. Evol. Microbiol. 38:89-98.

Fernández-López, M., Goormachtig, S., Gao, M., D’Haeze, W., Van Montagu, M., and Holsters, M. 1998. Ethylene-mediated phenotypic plasticity in root nodule development on Sesbania rostrata. Proc. Natl. Acad. Sci. U.S.A. 95:12724-12728.

Gao, M., D'Haeze, W., De Rycke, R., Wolucka, B., and Holsters, M. 2001. Knockout of an azorhizobial dTDP-L-rhamnose synthase affects lipopolysaccharide and extracellular polysaccharide production and disables symbiosis with Sesbania rostrata. Mol. Plant-Microbe Interact. 14:857-866.

Goethals, K., Leyman, B., Van Den Eede, G., Van Montagu, M., and Holsters, M. 1994. An Azorhizobium caulinodans ORS571 locus involved in lipopolysaccharide production and nodule formation on Sesbania rostrata stems and roots. J. Bacteriol. 176:92-99.

Goormachtig, S., Capoen, W., James, E. K., and Holsters, M. 2004. Switch from intracellular to intercellular invasion during water stress-tolerant legume nodulation. Proc. Natl. Acad. Sci. U.S.A. 101:6303-6308.

Hickman, J. W., Tifrea, D. F., and Harwood, C. S. 2005. A chemosensory system that regulates biofilm formation through modulation of cyclic diguanylate levels. Proc. Natl. Acad. Sci. U.S.A. 102:14422-14427.

Hirsch, A. M. 1992. Developmental biology of legume nodulation. New Phytol. 122:211-237.

Huang, Z., Wang, Y. H., Zhu, H. Z., Andrianova, E. P., Jiang, C. Y., Li, D., Ma, L., Feng, J., Liu, Z. P., Xiang, H., Zhulin, I. B., and Liu, S. J. 2019. Cross talk between chemosensory pathways that modulate chemotaxis and biofilm formation. MBio 10:e2876-18.

Huangyutitham, V., Güvener, Z. T., and Harwood, C. S. 2013. Subcellular clustering of the phosphorylated WspR response regulator protein stimulates its diguanylate cyclase activity. MBio 4:e00242-13.

Janczarek, M., Rachwal, K., Ciesla, J., Ginalska, G., and Bieganowski, A. 2015. Production of exopolysaccharide by Rhizobium leguminosarum bv. trifolii and its role in bacterial attachment and surface properties. Plant Soil 388:211-227. 
Jiang, N., Liu, W., Li, Y., Wu, H., Zhang, Z., Alexandre, G., Elmerich, C., and Xie, Z. 2016. A Chemotaxis receptor modulates nodulation during the Azorhizobium caulinodans-Sesbania rostrata symbiosis. Appl. Environ. Microbiol. 82:3174-3184.

Jofré, E., Lagares, A., and Mori, G. 2004. Disruption of dTDP-rhamnose biosynthesis modifies lipopolysaccharide core, exopolysaccharide production, and root colonization in Azospirillum brasilense. FEMS Microbiol. Lett. 231:267-275.

Jones, C. W., and Armitage, J. P. 2015. Positioning of bacterial chemoreceptors. Trends Microbiol. 23:247-256.

Kamilova, F., Kravchenko, L. V., Shaposhnikov, A. I., Azarova, T., Makarova, N., and Lugtenberg, B. 2006. Organic acids, sugars, and L-tryptophane in exudates of vegetables growing on stonewool and their effects on activities of rhizosphere bacteria. Mol. Plant-Microbe Interact. 19:250-256.

Kirby, J. R., and Zusman, D. R. 2003. Chemosensory regulation of developmental gene expression in Myxococcus xanthus. Proc. Natl. Acad. Sci. U.S.A. 100:2008-2013.

Lacal, J., Alfonso, C., Liu, X., Parales, R. E., Morel, B., Conejero-Lara, F., Rivas, G., Duque, E., Ramos, J. L., and Krell, T. 2010. Identification of a chemoreceptor for tricarboxylic acid cycle intermediates: Differential chemotactic response towards receptor ligands. J. Biol. Chem. 285: 23126-23136.

Liu, H., Wang, X., Qi, H., Wang, Q., Chen, Y., Li, Q., Zhang, Y., Qiu, L., Fontana, J. E., Zhang, B., Wang, W., and Xie, Y. 2017. The infection and impact of Azorhizobium caulinodans ORS571 on wheat (Triticum aestivum L.). PLoS One 12:e0187947.

Liu, W., Sun, Y., Shen, R., Dang, X., Liu, X., Sui, F., Li, Y., Zhang, Z., Alexandre, G., Elmerich, C., and Xie, Z. 2018. A chemotaxis-like pathway of Azorhizobium caulinodans controls flagella-driven motility, which regulates biofilm formation, exopolysaccharide biosynthesis, and competitive nodulation. Mol. Plant-Microbe Interact. 31:737-749.

Liu, W., Yang, J., Sun, Y., Liu, X., Li, Y., Zhang, Z., and Xie, Z. 2017. Azorhizobium caulinodans transmembrane chemoreceptor TlpA1 involved in host colonization and nodulation on roots and stems. Front. Microbiol. 8:1327.

Liu, X., Liu, W., Sun, Y., Xia, C., Elmerich, C., and Xie, Z. 2018. A cheZlike gene in Azorhizobium caulinodans is a key gene in the control of chemotaxis and colonization of the host plant. Appl. Environ. Microbiol. 84:e01827-17.

Ma, L., Jackson, K. D., Landry, R. M., Parsek, M. R., and Wozniak, D. J. 2006. Analysis of Pseudomonas aeruginosa conditional psl variants reveals roles for the psl polysaccharide in adhesion and maintaining biofilm structure postattachment. J. Bacteriol. 188:8213-8221.

Marx, C. J., and Lidstrom, M. E. 2002. Broad-host-range cre-lox system for antibiotic marker recycling in gram-negative bacteria. Biotechniques 33: 1062-1067.

Mathis, R., Van Gijsegem, F., De Rycke, R., D’Haeze, W., Van Maelsaeke, E., Anthonio, E., Van Montagu, M., Holsters, M., and Vereecke, D. 2005. Lipopolysaccharides as a communication signal for progression of legume endosymbiosis. Proc. Natl. Acad. Sci. U.S.A. 102:2655-2660.

McGee, D. J., Langford, M. L., Watson, E. L., Carter, J. E., Chen, Y. T., and Ottemann, K. M. 2005. Colonization and inflammation deficiencies in Mongolian gerbils infected by Helicobacter pylori chemotaxis mutants. Infect. Immun. 73:1820-1827.

Mendis, H. C., Madzima, T. F., Queiroux, C., and Jones, K. M. 2016. Function of succinoglycan polysaccharide in Sinorhizobium meliloti host plant invasion depends on succinylation, not molecular weight. MBio 7: e00606-16.

Miller, L. D., Yost, C. K., Hynes, M. F., and Alexandre, G. 2007. The major chemotaxis gene cluster of Rhizobium leguminosarum bv. viciae is essential for competitive nodulation. Mol. Microbiol. 63:348-362.
Mitra, S., Mukherjee, A., Wiley-Kalil, A., Das, S., Owen, H., Reddy, P. M., Ané, J. M., James, E. K., and Gyaneshwar, P. 2016. A rhamnosedeficient lipopolysaccharide mutant of Rhizobium sp. IRBG74 is defective in root colonization and beneficial interactions with its flooding-tolerant hosts Sesbania cannabina and wetland rice. J. Exp. Bot. 67:5869-5884

Mongiardini, E. J., Ausmees, N., Pérez-Giménez, J., Althabegoiti, M. J., Quelas, J. I., López-García, S. L., and Lodeiro, A. R. 2008. The rhizobial adhesion protein RapA1 is involved in adsorption of rhizobia to plant roots but not in nodulation. FEMS Microbiol. Ecol. 65:279-288.

Nakajima, A., Aono, T., Tsukada, S., Siarot, L., Ogawa, T., and Oyaizu, H. 2012. Lon protease of Azorhizobium caulinodans ORS571 is required for suppression of $r e b$ gene expression. Appl. Environ. Microbiol. 78: 6251-6261

Ndoye, I., de Billy, F., Vasse, J., Dreyfus, B., and Truchet, G. 1994. Root nodulation of Sesbania rostrata. J. Bacteriol. 176:1060-1068.

Qiu, L., Lin, J. S., Xu, J., Sato, S., Parniske, M., Wang, T. L., Downie, J. A., and Xie, F. 2015. SCARN a novel class of SCAR protein that is required for root-hair infection during legume nodulation. PLoS Genet. 11: e1005623.

Rivas, R., Velázquez, E., Willems, A., Vizcaíno, N., Subba-Rao, N. S., Mateos, P. F., Gillis, M., Dazzo, F. B., and Martínez-Molina, E. 2002. A new species of Devosia that forms a unique nitrogen-fixing root-nodule symbiosis with the aquatic legume Neptunia natans (L.f.) Druce. Appl. Environ. Microbiol. 68:5217-5222.

Scharf, B. E., Hynes, M. F., and Alexandre, G. M. 2016. Chemotaxis signaling systems in model beneficial plant-bacteria associations. Plant Mol. Biol. 90:549-559.

Schmittgen, T. D., and Livak, K. J. 2008. Analyzing real-time PCR data by the comparative C(T) method. Nat. Protoc. 3:1101-1108.

Sonnenschein, E. C., Syit, D. A., Grossart, H. P., and Ullrich, M. S. 2012. Chemotaxis of Marinobacter adhaerens and its impact on attachment to the diatom Thalassiosira weissflogii. Appl. Environ. Microbiol. 78: 6900-6907.

Terry, K., Williams, S. M., Connolly, L., and Ottemann, K. M. 2005. Chemotaxis plays multiple roles during Helicobacter pylori animal infection. Infect. Immun. 73:803-811.

Tsien, H. C., Dreyfus, B. L., and Schmidt, E. L. 1983. Initial stages in the morphogenesis of nitrogen-fixing stem nodules of Sesbania rostrata. J. Bacteriol. 156:888-897.

Tsukada, S., Aono, T., Akiba, N., Lee, K. B., Liu, C. T., Toyazaki, H., and Oyaizu, H. 2009. Comparative genome-wide transcriptional profiling of Azorhizobium caulinodans ORS571 grown under free-living and symbiotic conditions. Appl. Environ. Microbiol. 75:5037-5046.

Vallad, G. E., and Subbarao, K. V. 2008. Colonization of resistant and susceptible lettuce cultivars by a green fluorescent protein-tagged isolate of Verticillium dahliae. Phytopathology 98:871-885.

Wakao, S., Siarot, L., Aono, T., and Oyaizu, H. 2015. Effects of alteration in LPS structure in Azorhizobium caulinodans on nodule development. J. Gen. Appl. Microbiol. 61:248-254.

Willett, J. W., and Kirby, J. R. 2011. CrdS and CrdA comprise a twocomponent system that is cooperatively regulated by the Che 3 chemosensory system in Myxococcus xanthus. MBio 2:e00110-11.

Yao, J., and Allen, C. 2006. Chemotaxis is required for virulence and competitive fitness of the bacterial wilt pathogen Ralstonia solanacearum. J. Bacteriol. 188:3697-3708.

Yaryura, P. M., León, M., Correa, O. S., Kerber, N. L., Pucheu, N. L., and García, A. F. 2008. Assessment of the role of chemotaxis and biofilm formation as requirements for colonization of roots and seeds of soybean plants by Bacillus amyloliquefaciens BNM339. Curr. Microbiol. 56: 625-632. 